Article

\title{
Development and Validation of a HPLC-MS/MS Method for Simultaneous Determination of Twelve Bioactive Compounds in Epimedium: Application to a Pharmacokinetic Study in Rats
}

\author{
Mengjie Sun, Yanwei Yin, Juan Wei, Xiaopeng Chen, Huizi Ouyang, Yanxu Chang ${ }^{\mathbb{D}}$, Xiumei Gao \\ and Jun $\mathrm{He}^{*}$ \\ Tianjin State Key Laboratory of Modern Chinese Medicine, Tianjin University of Traditional Chinese Medicine, \\ 312 Anshanxi Road, Nankai District, Tianjin 300193, China; 15122865529@163.com (M.S.); \\ Yinyw8739@163.com (Y.Y.); 15222797261@163.com (J.W.); xpchen@tjutcm.edu.cn (X.C.); \\ huihui851025@163.com (H.O.); tcmcyx@126.com (Y.C.); gaoxiumei@tjutcm.edu.cn (X.G.) \\ * Correspondence: hejun673@tjutcm.edu.cn; Tel./Fax: +86-22-5959-6163
}

Received: 6 May 2018; Accepted: 28 May 2018; Published: 31 May 2018

\begin{abstract}
A rapid and reliable HPLC-MS/MS method has been developed and validated for the simultaneous quantification of twelve bioactive compounds (baohuoside II, baohuoside I, sagittatoside A, sagittatoside B, magnoflorine, epimedin A, epimedin B, epimedin C, chlorogenic acid, neochlorogenic acid, cryptochlorogenic acid and icariin) in rat plasma. The collected plasma samples were prepared by protein precipitate with acetonitrile. The twelve compounds were separated on a CORTECS ${ }^{\circledR} \mathrm{C} 18$ column $(4.6 \mathrm{~mm} \times 150 \mathrm{~mm}, 2.7 \mu \mathrm{m})$ with a gradient mobile phase system of $0.1 \%$ $(v / v)$ formic acid and acetonitrile at a flow rate of $0.3 \mathrm{~mL} / \mathrm{min}$. All of the analytes were quantitated using electrospray ionization (ESI) in negative ion mode with selected reaction monitoring (SRM). The intra- and inter-day accuracy ranged from $-5.6 \%$ to $13.0 \%$, and the precisions of the analytes were less than $10.9 \%$. The mean recoveries of the analytes were in the range of $60.66 \%$ to $99.77 \%$ and the matrix effect ranged from $93.08 \%$ to $119.84 \%$. Stability studies proved that the analytes were stable under the tested conditions, with a relative standard deviation (RSD) lower than $11.7 \%$. The developed method was successfully applied to evaluating the pharmacokinetic study of twelve bioactive compounds after oral administration of Epimedium extract in rat.
\end{abstract}

Keywords: Epimedium extract; pharmacokinetics; HPLC-MS/MS; rat plasma

\section{Introduction}

Epimedium is a traditional tonic herb used for "reinforcing the Yang and nourishing the kidney" in China. Four species of Epimedium, including Epimedium koreanum Nakai., Epimedium pubescens Maxim., Epimedium brevicornu Maxim. and Epimedium sagittatum (Sieb. et Zucc.) Maxim., are recorded in Chinese Pharmacopeia (Edition 2015). Traditionally, Epimedium are used to treat impotence, forgetfulness and neurasthenia [1]. Nowadays, pharmacological study has demonstrated that Epimedium possesses a variety of activities, such as inhibition of tumor, anti-oxidation and anti-inflammatory [2,3]. Meanwhile, clinical research has suggested the treatment of osteoporosis, diabetes, releasing depression, cardiovascular diseases, chronic nephritis and rheumatoid arthritis with Epimedium [4-7].

Phytochemical investigation has found many kinds of compounds isolated and identified from Epimedium, such as flavonoid glycosides, phenylpropanoids, alkaloids, polysaccharides, lignins and sesquiterpenes [8]. The most important active compounds are flavonoid glycosides, including 
icariin, icariside-II, epimedin A and epimedin B, etc. [9]. The flavonoid glycosides show the activity in treatment of inflammatory, metabolic disorder, osteoporosis, diabetes and cancer [10-13]. Phenylpropanoids including chlorogenic acid, neochlorogenic acid and cryptochlorogenic acid have anti-inflammatory, anti-oxidation and anti-cancer effects [14]. Magnoflorine, the alkaloid from Epimedium, displays hypoglycemic activity [15]. In addition, polysaccharides and their chemical derivatives from Epimedium, such as glucose, rhamnose and mannose, possess immunomodulatory, anti-tumor, anti-oxidation, and anti-microbial activities [16,17].

Pharmacokinetic study plays an important role in clinical applications with regard to determination of the clinical dose and avoiding side effects. Several manuscripts focusing on the pharmacokinetics of Epimedium have been published, such as the validation of an LC-MS/MS method to quantify five flavonoid glycosides (icariin, icaritin, desmethylicaritin, icariside I and icariside II) simultaneously in Epimedium [18]. Furthermore, the pharmacokinetic properties of icariin, epimedin A, epimedin B, epimedin C, baohuoside I, sagittatoside B and 2" -O-rhamnosyl icariside II have been determined in dogs in one injection [19].

In this study, a reliable HPLC-MS/MS method was developed to quantify twelve bioactive compounds (baohuoside I, baohuoside II, sagittatoside A, sagittatoside B, magnoflorine, epimedin A, epimedin B, epimedin C, chlorogenic acid, neochlorogenic acid, cryptochlorogenic acid and icariin) simultaneously in rat plasma. Then the assay was applied to the pharmacokinetic study of the compounds in rats after oral administration of Epimedium extract.

\section{Results and Discussion}

\subsection{Method Development}

To obtain a better separation and a shorter retention time, a variety of mobile phases such as acetonitrile-water, acetonitrile- $0.1 \%(v / v)$ formic acid, methanol-water and methanol- $0.1 \%(v / v)$ formic acid were tested. Finally, acetonitrile- $0.1 \%(v / v)$ formic acid was used as the optimum mobile phase on CORTECS ${ }^{\circledR} \mathrm{C} 18$ column. As shown in Figure 1, 12 analytes and IS were eluted for $15 \mathrm{~min}$, and no interfering peaks were observed.

To optimize the mass spectrometry conditions, negative and positive modes were tested. The negative mode showed better intensity for analytes and lower background than the positive mode. In addition, the capillary temperature, auxiliary nitrogen pressure, drying gas flow and ion spray voltage were optimized to obtain the most suitable electrospray ionization parameters as described in Section 3.2.

\subsection{Sample Preparation}

To develop a simple and efficient sample preparation method, liquid-liquid extraction and protein precipitation were tested. Liquid-liquid extraction using ethyl acetate achieved good recovery for flavonoid glycosides, but not for chlorogenic acid, neochlorogenic acid and cryptochlorogenic acid. Protein precipitation with methanol and acetonitrile were compared, and acetonitrile precipitation showed better recovery. Moreover, to obtain optimum extraction, different kinds of acids (formic acid, acetic acid and phosphoric acid) were added to acetonitrile and compared. In consideration of everything, the protein precipitation using acetonitrile containing formic acid was used in the sample preparation for all analytes.

\subsection{Method Validation}

\subsubsection{Specificity}

Blank plasma samples were prepared as described in Section 3.5. The chromatograms of blank plasma (A), blank plasma samples spiked with analytes and IS (B), and plasma samples collected 
15 min after oral administration of Epimedium extract $(C)$ are shown in Figure 1. The results show that no interfering peaks were observed in the samples.

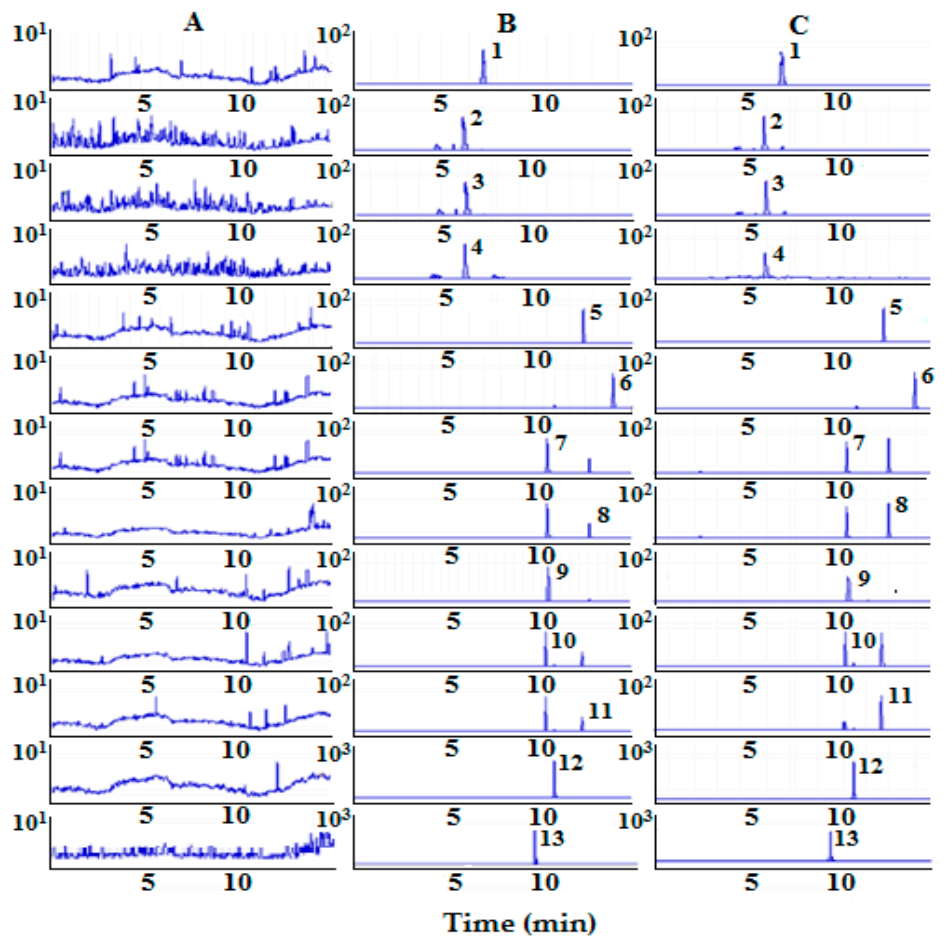

Figure 1. SRM chromatograms of magnoflorine (1), neochlorogenic acid (2), chlorogenic acid (3), cryptochlorogenic acid (4), baohuoside II (5), baohuoside I (6), epimedin B (7), sagittatoside B (8), epimedin C (9), epimedin A (10), sagittatoside A (11), icariin (12) and IS (13). (A) Blank plasma; (B) blank plasma spiked with the analytes and IS; (C) plasma sample $15 \mathrm{~min}$ after oral administration of Epimedium extract.

\subsubsection{Calibration Curves}

Calibration curves and LLOQs in rat plasma are listed in Table 1. The regression coefficient (r) was greater than 0.9904 for all calibration curves. This demonstrates that the analytes have good linearity over the linear range. The LLOQs of the 12 analytes were less than $10 \mathrm{ng} / \mathrm{mL}$.

\subsubsection{Precision and Accuracy}

The precision and accuracy of the 12 analytes is summarized in Table 2. As shown in the table, the intra- and inter-day accuracy (RE) ranged from -5.6 to $12.9 \%$, and the precision (RSD) ranged from $0.4 \%$ to $10.9 \%$. The results prove that the developed method is accurate and precise.

\subsubsection{Extraction Recovery and Matrix Effect}

The extraction recoveries and matrix effect of QC samples at three different concentrations are summarized in Table 3. The extraction recoveries of QC samples were in the range of $60.66 \%$ to $99.77 \%$, with RSDs of less than $11.3 \%$. The matrix effect ranged from $93.08 \%$ to $119.84 \%$, with RSDs of less than $13.2 \%$. The results show that the extraction recovery and the matrix effect are acceptable.

\subsubsection{Stability}

The stability of QC samples was summarized in Table 4. The RSD of the replicate QC samples was less than $11.7 \%$. The data demonstrates that the analytes are stable at room temperature for $2 \mathrm{~h}$, in an auto-sampler for $12 \mathrm{~h}$, and for 7 days and through three freeze-thaw cycles at $-70{ }^{\circ} \mathrm{C}$. 


\subsection{Application}

The plasma samples obtained from the rats to which Epimedium extract had been administrated by gavage were determined by the HPLC-MS/MS method. Plasma concentration-time curves of twelve compounds in rats are shown in Figure 2, and the major pharmacokinetic parameters are depicted in Table 5.

According to the pharmacokinetic parameters, the analytes were divided into two groups: one group includes baohuoside I, baohuoside II, sagittatoside A, sagittatoside B, magnoflorine, epimedin A, epimedin B and epimedin C (group A), and the other group includes chlorogenic acid, neochlorogenic acid, cryptochlorogenic acid and icariin (group B). A bimodal phenomenon appeared in the plasma concentration-time curves of the analytes of group A. The $T_{\max 1}$ of baohuoside I, baohuoside II, sagittatoside A, sagittatoside B, magnoflorine, epimedin A, epimedin B and epimedin C in group A were $0.38 \pm 0.14 \mathrm{~h}, 0.18 \pm 0.03 \mathrm{~h}, 0.18 \pm 0.03 \mathrm{~h}, 0.20 \pm 0.04 \mathrm{~h}, 0.18 \pm 0.03 \mathrm{~h}, 0.22 \pm 0.04 \mathrm{~h}, 0.19 \pm 0.07 \mathrm{~h}$ and $0.22 \pm 0.04 \mathrm{~h}$, respectively, and the $T_{\max 2}$ were $10.00 \pm 1.79 \mathrm{~h}, 10.67 \pm 1.03 \mathrm{~h}, 11.67 \pm 0.82 \mathrm{~h}$, $11.00 \pm 1.67 \mathrm{~h}, 10.67 \pm 1.63 \mathrm{~h}, 9.33 \pm 0.94 \mathrm{~h}, 7.67 \pm 0.75 \mathrm{~h}$ and $7.67 \pm 0.75 \mathrm{~h}$, respectively. This bimodal phenomenon of the analytes (epimedin A, epimedin B and baohuoside I) was also found in previous reports [19]. This phenomenon may be caused by glucuronidation or enterohepatic circulation [20]. The $T_{\max }$ of chlorogenic acid, neochlorogenic acid, cryptochlorogenic acid and icariin in group $\mathrm{B}$ was $0.19 \pm 0.05 \mathrm{~h}, 0.19 \pm 0.05 \mathrm{~h}, 0.19 \pm 0.05 \mathrm{~h}$ and $0.16 \pm 0.05 \mathrm{~h}$, respectively, which is coincident with the reported literature [21,22]. The $t_{1 / 2}$ of group A and B ranged from $3 \mathrm{~h}$ to $18 \mathrm{~h}$ and $0.5 \mathrm{~h}$ to $1 \mathrm{~h}$, respectively. The results indicated that the analytes of group $\mathrm{B}$ were absorbed and eliminated quickly in the rat plasma after oral administration of Epimedium extract. Meanwhile, the analytes of group A required a long time to eliminate after absorption.

Table 1. Calibration curves, correlation coefficients, linear ranges and LLOQ of the analytes.

\begin{tabular}{ccccc}
\hline Compounds & Calibration Curve & $\mathbf{r}$ & Linear Range (ng/mL) & LLOQ(ng/mL) \\
\hline baohuoside II & $\mathrm{Y}=1.3944 \mathrm{X}+4.6850$ & 0.9930 & $1.0-200.0$ & 1.0 \\
baohuoside I & $\mathrm{Y}=1.4452 \mathrm{X}+0.0129$ & 0.9943 & $2.0-400.0$ & 2.0 \\
sagittatoside A & $\mathrm{Y}=0.5707 \mathrm{X}+4.8545$ & 0.9918 & $2.0-400.0$ & 2.0 \\
sagittatoside B & $\mathrm{Y}=0.6968 X+7.8080$ & 0.9908 & $2.0-400.0$ & 2.0 \\
epimedin A & $\mathrm{Y}=0.1564 \mathrm{X}-8.1949$ & 0.9912 & $2.0-400.0$ & 2.0 \\
epimedin B & $\mathrm{Y}=0.3559 \mathrm{X}+5.3440$ & 0.9939 & $2.0-400.0$ & 2.0 \\
epimedin C & $\mathrm{Y}=0.1016 \mathrm{X}+6.8756$ & 0.9942 & $2.0-400.0$ & 4.0 \\
magnoflorine & $\mathrm{Y}=0.0260 \mathrm{X}+2.4814$ & 0.9928 & $4.0-800.0$ & 10.0 \\
chlorogenic acid & $\mathrm{Y}=0.1274 \mathrm{X}+0.0016$ & 0.9922 & $10.0-2000.0$ & 4.0 \\
neochlorogenic acid & $\mathrm{Y}=1.7722 \mathrm{X}+0.0013$ & 0.9956 & $4.0-800.0$ & 4.0 \\
cryptochlorogenic acid & $\mathrm{Y}=0.0187 \mathrm{X}+0.0010$ & 0.9904 & $4.0-800.0$ & 4.0 \\
icariin & $\mathrm{Y}=0.1178 \mathrm{X}+0.0053$ & 0.9966 & $4.0-800.0$ & \\
\hline
\end{tabular}

Table 2. Precision and accuracy of 12 analytes in rat plasma $(n=6)$.

\begin{tabular}{|c|c|c|c|c|c|c|c|}
\hline \multirow[b]{2}{*}{ Compounds } & \multirow{2}{*}{$\begin{array}{l}\text { Spiked Concentration } \\
(\mathrm{ng} / \mathrm{mL})\end{array}$} & \multicolumn{3}{|c|}{ Intra-Day } & \multicolumn{3}{|c|}{ Inter-Day } \\
\hline & & $\begin{array}{c}\text { Measured } \\
\text { (ng/mL) }\end{array}$ & RE (\%) & RSD (\%) & $\begin{array}{l}\text { Measured } \\
\text { (ng/mL) }\end{array}$ & RE (\%) & RSD (\%) \\
\hline \multirow{3}{*}{ baohuoside II } & 1 & $1.02 \pm 0.08$ & 2.0 & 7.8 & $1.01 \pm 0.08$ & 1.0 & 7.9 \\
\hline & 10 & $10.18 \pm 0.88$ & 1.8 & 8.6 & $10.09 \pm 0.87$ & 0.9 & 8.6 \\
\hline & 200 & $197.54 \pm 1.41$ & -1.2 & 0.7 & $196.66 \pm 3.62$ & -1.7 & 1.8 \\
\hline \multirow{3}{*}{ baohuoside I } & 2 & $2.03 \pm 0.07$ & 1.5 & 3.4 & $2.03 \pm 0.08$ & 1.5 & 3.9 \\
\hline & 20 & $19.99 \pm 0.98$ & -0.1 & 4.9 & $19.54 \pm 0.92$ & -2.3 & 4.7 \\
\hline & 400 & $390.27 \pm 6.47$ & -2.4 & 1.7 & $391.05 \pm 7.15$ & -2.2 & 1.8 \\
\hline \multirow{3}{*}{ sagittatoside A } & 2 & $2.06 \pm 0.10$ & 3.0 & 4.9 & $1.98 \pm 0.12$ & -1.0 & 6.0 \\
\hline & 20 & $20.77 \pm 1.01$ & 3.9 & 4.9 & $21.00 \pm 0.90$ & 5.0 & 4.3 \\
\hline & 400 & $399.42 \pm 5.65$ & -0.1 & 1.4 & $396.13 \pm 3.57$ & -1.0 & 0.9 \\
\hline \multirow{3}{*}{ sagittatoside B } & 2 & $2.08 \pm 0.06$ & 4.0 & 2.9 & $2.14 \pm 0.07$ & 7.0 & 3.3 \\
\hline & 20 & $20.50 \pm 1.36$ & 2.5 & 6.6 & $21.07 \pm 0.81$ & 5.4 & 3.8 \\
\hline & 400 & $397.48 \pm 7.79$ & -0.6 & 2.0 & $404.06 \pm 7.29$ & 1.0 & 1.8 \\
\hline
\end{tabular}


Table 2. Cont.

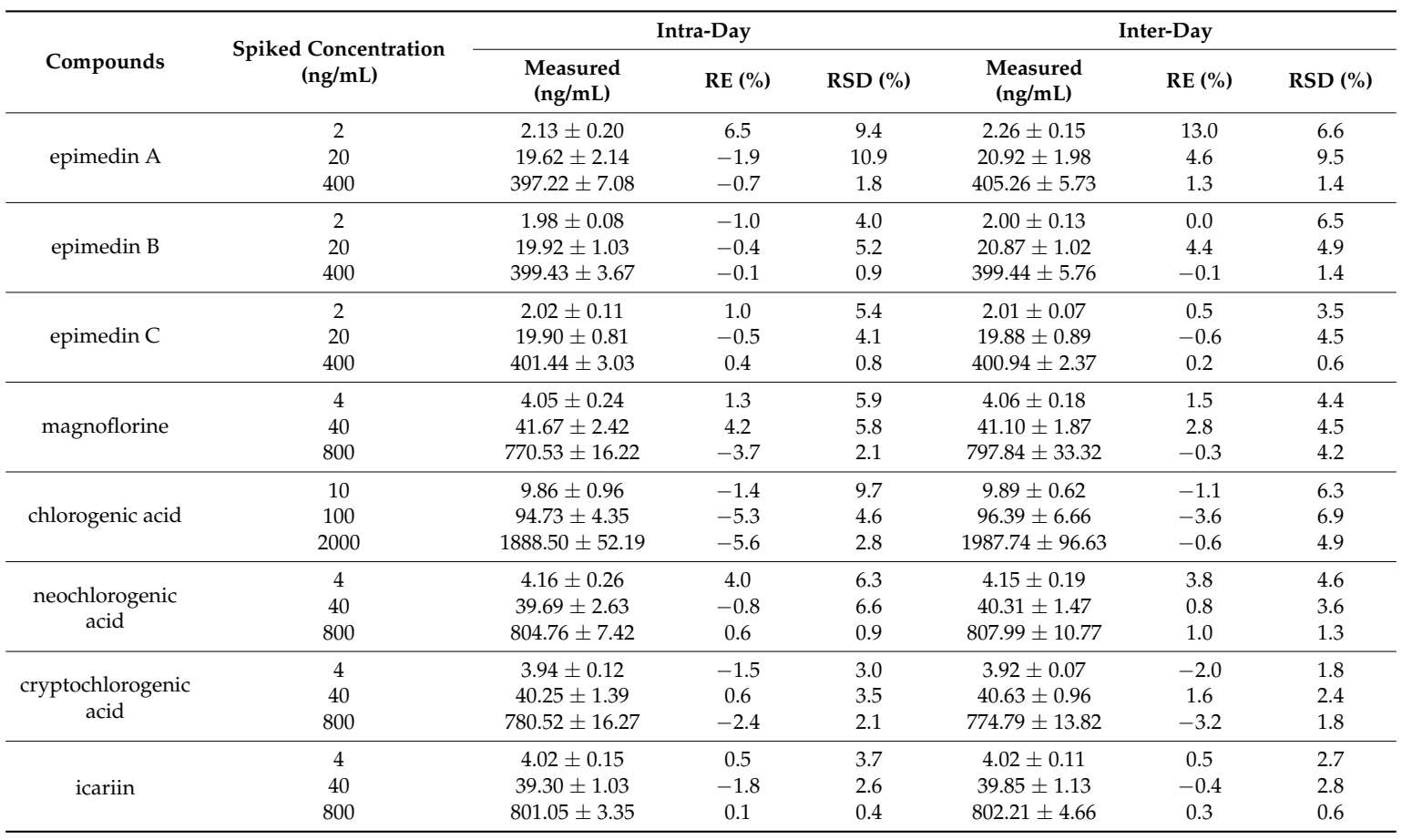

Table 3. Extraction recoveries and matrix effects of the analytes $(n=6)$.

\begin{tabular}{|c|c|c|c|c|c|}
\hline Compounds & Spiked Concentration (ng/mL) & Extraction Recovery (\%) & RSD (\%) & Matrix Effect (\%) & RSD (\%) \\
\hline \multirow{3}{*}{ baohuoside II } & 1 & $67.78 \pm 1.75$ & 2.6 & $119.03 \pm 3.54$ & 3.0 \\
\hline & 10 & $84.44 \pm 3.81$ & 4.5 & $115.54 \pm 6.71$ & 5.8 \\
\hline & 200 & $93.58 \pm 4.14$ & 4.4 & $103.39 \pm 7.55$ & 7.3 \\
\hline \multirow{3}{*}{ baohuoside I } & 2 & $99.45 \pm 6.74$ & 6.8 & $93.08 \pm 5.89$ & 6.3 \\
\hline & 20 & $97.78 \pm 1.36$ & 1.4 & $103.85 \pm 6.55$ & 6.3 \\
\hline & 400 & $98.50 \pm 1.73$ & 1.8 & $98.21 \pm 3.20$ & 3.3 \\
\hline \multirow{3}{*}{ sagittatoside A } & 2 & $74.52 \pm 7.02$ & 9.4 & $112.96 \pm 8.54$ & 7.6 \\
\hline & 20 & $79.30 \pm 3.64$ & 4.6 & $118.72 \pm 9.92$ & 8.4 \\
\hline & 400 & $90.06 \pm 3.35$ & 3.7 & $107.33 \pm 10.01$ & 9.3 \\
\hline \multirow{3}{*}{ sagittatoside B } & 2 & $99.77 \pm 4.19$ & 4.2 & $98.77 \pm 3.97$ & 4.0 \\
\hline & 20 & $82.34 \pm 4.82$ & 5.9 & $112.35 \pm 7.63$ & 6.8 \\
\hline & 400 & $86.73 \pm 2.69$ & 3.1 & $111.49 \pm 6.28$ & 5.6 \\
\hline \multirow{3}{*}{ epimedin A } & 2 & $60.66 \pm 6.63$ & 10.9 & $113.02 \pm 10.87$ & 9.6 \\
\hline & 20 & $68.52 \pm 0.66$ & 1.0 & $119.11 \pm 13.22$ & 11.1 \\
\hline & 400 & $84.85 \pm 3.63$ & 4.3 & $112.83 \pm 9.14$ & 8.1 \\
\hline \multirow{3}{*}{ epimedin B } & 2 & $70.66 \pm 4.43$ & 6.3 & $119.84 \pm 6.79$ & 5.7 \\
\hline & 20 & $94.72 \pm 0.75$ & 0.8 & $107.49 \pm 8.41$ & 7.8 \\
\hline & 400 & $75.30 \pm 1.12$ & 1.5 & $119.30 \pm 3.05$ & 2.6 \\
\hline \multirow{3}{*}{ epimedin $C$} & 2 & $96.35 \pm 1.00$ & 1.0 & $102.59 \pm 3.00$ & 2.9 \\
\hline & 20 & $70.91 \pm 2.30$ & 3.2 & $112.31 \pm 11.51$ & 10.2 \\
\hline & 400 & $81.57 \pm 1.92$ & 2.4 & $118.74 \pm 6.52$ & 5.5 \\
\hline \multirow{3}{*}{ magnoflorine } & 4 & $61.95 \pm 6.86$ & 11.1 & $115.27 \pm 15.25$ & 13.2 \\
\hline & 40 & $70.29 \pm 4.68$ & 6.7 & $114.36 \pm 5.95$ & 5.2 \\
\hline & 800 & $81.33 \pm 4.59$ & 5.6 & $110.84 \pm 12.78$ & 11.5 \\
\hline \multirow{3}{*}{ chlorogenic acid } & 10 & $97.79 \pm 4.36$ & 4.5 & $98.55 \pm 7.49$ & 7.6 \\
\hline & 100 & $89.78 \pm 8.65$ & 9.6 & $98.36 \pm 5.32$ & 5.4 \\
\hline & 2000 & $78.81 \pm 5.22$ & 6.6 & $114.50 \pm 13.84$ & 12.1 \\
\hline \multirow{3}{*}{ neochlorogenic acid } & 4 & $82.92 \pm 2.58$ & 3.1 & $113.90 \pm 5.98$ & 5.3 \\
\hline & 40 & $78.53 \pm 2.88$ & 3.7 & $114.36 \pm 5.95$ & 5.2 \\
\hline & 800 & $84.04 \pm 3.36$ & 4.0 & $115.44 \pm 8.99$ & 7.8 \\
\hline \multirow{3}{*}{ cryptochlorogenic acid } & 4 & $91.38 \pm 10.31$ & 11.3 & $96.33 \pm 6.89$ & 7.2 \\
\hline & 40 & $76.05 \pm 6.75$ & 8.9 & $118.92 \pm 11.70$ & 9.8 \\
\hline & 800 & $76.95 \pm 3.26$ & 4.2 & $116.51 \pm 12.06$ & 10.4 \\
\hline \multirow{3}{*}{ icariin } & 4 & $96.32 \pm 4.65$ & 4.8 & $95.68 \pm 5.32$ & 5.6 \\
\hline & 40 & $88.84 \pm 9.11$ & 10.3 & $111.87 \pm 10.96$ & 9.8 \\
\hline & 800 & $91.78 \pm 4.42$ & 4.8 & $105.94 \pm 8.98$ & 8.5 \\
\hline
\end{tabular}


Table 4. Stability of all analytes in rat plasma $(n=3)$.

\begin{tabular}{|c|c|c|c|c|c|c|c|c|c|}
\hline \multirow{2}{*}{ Compounds } & \multirow{2}{*}{ Spiked Concentration $(\mathrm{ng} / \mathrm{mL})$} & \multicolumn{2}{|c|}{ Room Temperature for $2 \mathrm{~h}$} & \multicolumn{2}{|c|}{ Three Freeze-Thaw Cycles } & \multicolumn{2}{|c|}{ Auto-Sampler for $12 \mathrm{~h}$} & \multicolumn{2}{|c|}{$-70^{\circ} \mathrm{C}$ for 7 Days } \\
\hline & & Measured (ng/mL) & RSD (\%) & Measured (ng/mL) & RSD (\%) & Measured $(\mathrm{ng} / \mathrm{mL})$ & RSD (\%) & Measured (ng/mL) & RSD (\%) \\
\hline \multirow{3}{*}{ baohuoside II } & 1 & $0.96 \pm 0.09$ & 9.4 & $1.09 \pm 0.02$ & 1.8 & $1.10 \pm 0.11$ & 10.0 & $1.04 \pm 0.12$ & 11.5 \\
\hline & 10 & $9.99 \pm 0.31$ & 3.1 & $8.94 \pm 0.63$ & 7.0 & $10.38 \pm 0.60$ & 5.8 & $8.84 \pm 0.31$ & 3.5 \\
\hline & 200 & $193.54 \pm 3.74$ & 1.9 & $195.26 \pm 1.32$ & 0.7 & $190.62 \pm 5.99$ & 3.1 & $188.79 \pm 7.35$ & 3.9 \\
\hline \multirow{3}{*}{ baohuoside I } & 2 & $1.93 \pm 0.03$ & 1.6 & $1.97 \pm 0.08$ & 4.1 & $2.01 \pm 0.07$ & 3.5 & $2.03 \pm 0.09$ & 4.4 \\
\hline & 20 & $19.56 \pm 0.23$ & 1.2 & $19.71 \pm 0.49$ & 2.5 & $19.24 \pm 0.96$ & 5.0 & $19.48 \pm 0.52$ & 2.7 \\
\hline & 400 & $389.39 \pm 7.29$ & 1.9 & $393.71 \pm 5.02$ & 1.3 & $387.09 \pm 9.42$ & 2.4 & $391.78 \pm 3.49$ & 0.9 \\
\hline \multirow{3}{*}{ sagittatoside A } & 2 & $2.08 \pm 0.12$ & 5.8 & $2.11 \pm 0.07$ & 3.3 & $1.93 \pm 0.09$ & 4.7 & $1.98 \pm 0.12$ & 6.1 \\
\hline & 20 & $20.97 \pm 0.09$ & 0.4 & $19.84 \pm 0.81$ & 4.1 & $21.29 \pm 0.18$ & 0.8 & $19.43 \pm 0.40$ & 2.1 \\
\hline & 400 & $397.27 \pm 8.71$ & 2.2 & $393.69 \pm 3.71$ & 0.9 & $397.42 \pm 6.71$ & 1.7 & $395.24 \pm 11.34$ & 2.9 \\
\hline \multirow{3}{*}{ sagittatoside B } & 2 & $2.04 \pm 0.16$ & 7.8 & $2.09 \pm 0.05$ & 2.4 & $2.07 \pm 0.08$ & 3.9 & $2.03 \pm 0.14$ & 6.9 \\
\hline & 20 & $21.32 \pm 0.59$ & 2.8 & $20.07 \pm 0.95$ & 4.7 & $21.16 \pm 0.51$ & 2.4 & $18.84 \pm 0.52$ & 2.8 \\
\hline & 400 & $398.03 \pm 9.83$ & 2.5 & $394.07 \pm 3.55$ & 0.9 & $407.64 \pm 17.60$ & 4.3 & $381.68 \pm 5.87$ & 1.5 \\
\hline \multirow{3}{*}{ epimedin A } & 2 & $2.10 \pm 0.09$ & 4.3 & $2.07 \pm 0.13$ & 6.3 & $2.06 \pm 0.04$ & 1.9 & $2.03 \pm 0.09$ & 4.4 \\
\hline & 20 & $20.29 \pm 0.43$ & 2.1 & $19.58 \pm 0.94$ & 4.8 & $20.35 \pm 0.78$ & 3.8 & $19.70 \pm 1.54$ & 7.8 \\
\hline & 400 & $400.51 \pm 0.52$ & 0.1 & $392.00 \pm 2.85$ & 0.7 & $398.73 \pm 3.31$ & 0.8 & $400.95 \pm 4.79$ & 1.2 \\
\hline \multirow{3}{*}{ epimedin B } & 2 & $2.01 \pm 0.04$ & 2.0 & $2.07 \pm 0.09$ & 4.3 & $2.18 \pm 0.05$ & 2.3 & $2.07 \pm 0.09$ & 4.3 \\
\hline & 20 & $21.51 \pm 0.97$ & 4.5 & $20.27 \pm 1.82$ & 9.0 & $21.01 \pm 0.50$ & 2.4 & $21.00 \pm 0.78$ & 3.7 \\
\hline & 400 & $400.89 \pm 3.17$ & 0.8 & $400.51 \pm 2.96$ & 0.7 & $397.07 \pm 2.81$ & 0.7 & $403.31 \pm 2.82$ & 0.7 \\
\hline \multirow{3}{*}{ epimedin C } & 2 & $2.10 \pm 0.05$ & 2.4 & $2.03 \pm 0.10$ & 4.9 & $2.02 \pm 0.14$ & 6.9 & $2.14 \pm 0.05$ & 2.3 \\
\hline & 20 & $20.24 \pm 0.83$ & 4.1 & $20.43 \pm 0.90$ & 4.4 & $19.73 \pm 0.37$ & 1.9 & $21.37 \pm 0.10$ & 0.5 \\
\hline & 400 & $399.97 \pm 1.97$ & 0.5 & $397.64 \pm 8.12$ & 2.0 & $396.56 \pm 4.69$ & 1.2 & $396.46 \pm 8.82$ & 2.2 \\
\hline \multirow{3}{*}{ magnoflorine } & 4 & $3.99 \pm 0.08$ & 2.0 & $4.01 \pm 0.13$ & 3.2 & $4.37 \pm 0.19$ & 4.3 & $4.02 \pm 0.10$ & 2.5 \\
\hline & 40 & $39.29 \pm 2.92$ & 7.4 & $41.99 \pm 2.30$ & 5.5 & $41.70 \pm 1.06$ & 2.5 & $40.27 \pm 1.70$ & 4.2 \\
\hline & 800 & $776.84 \pm 1.85$ & 0.2 & $780.80 \pm 15.38$ & 2.0 & $809.27 \pm 12.34$ & 1.5 & $775.71 \pm 3.82$ & $\begin{array}{l}4.2 \\
0.5\end{array}$ \\
\hline \multirow{3}{*}{ chlorogenic acid } & 10 & $9.93 \pm 0.03$ & 0.3 & $9.52 \pm 0.84$ & 8.8 & $9.26 \pm 0.68$ & 7.3 & $8.88 \pm 0.39$ & 4.4 \\
\hline & 100 & $101.51 \pm 11.83$ & 11.7 & $91.99 \pm 6.26$ & 6.8 & $98.92 \pm 4.58$ & 4.6 & $85.73 \pm 4.01$ & 4.7 \\
\hline & 2000 & $1866.65 \pm 25.87$ & 1.4 & $1883.55 \pm 25.34$ & 1.3 & $1924.54 \pm 82.95$ & 4.3 & $1849.43 \pm 30.88$ & 1.7 \\
\hline \multirow{3}{*}{ neochlorogenic acid } & 4 & $4.00 \pm 0.14$ & 3.5 & $3.97 \pm 0.05$ & 1.3 & $3.85 \pm 0.14$ & 3.6 & $4.13 \pm 0.06$ & 1.5 \\
\hline & 40 & $39.79 \pm 2.13$ & 5.4 & $38.73 \pm 1.14$ & 2.9 & $41.77 \pm 1.37$ & 3.3 & $39.14 \pm 1.82$ & 4.6 \\
\hline & 800 & $802.82 \pm 7.13$ & 0.9 & $791.74 \pm 0.87$ & 0.1 & $803.36 \pm 2.53$ & 0.3 & $804.80 \pm 4.45$ & 0.6 \\
\hline \multirow{3}{*}{ cryptochlorogenic acid } & 4 & $4.00 \pm 0.14$ & 3.5 & $4.03 \pm 0.13$ & 3.2 & $4.01 \pm 0.22$ & 5.5 & $4.01 \pm 0.06$ & 1.5 \\
\hline & 40 & $40.59 \pm 1.78$ & 4.4 & $39.42 \pm 1.80$ & 4.6 & $41.58 \pm 0.51$ & 1.2 & $39.06 \pm 1.65$ & 4.2 \\
\hline & 800 & $773.95 \pm 5.78$ & 0.7 & $781.42 \pm 2.45$ & 0.3 & $768.52 \pm 23.65$ & 3.1 & $759.75 \pm 7.73$ & 1.0 \\
\hline \multirow{3}{*}{ icariin } & 4 & $4.07 \pm 0.10$ & 2.5 & $4.09 \pm 0.09$ & 2.2 & $4.11 \pm 0.10$ & 2.4 & $4.00 \pm 0.06$ & 1.5 \\
\hline & 40 & $40.07 \pm 0.42$ & 1.0 & $40.45 \pm 1.98$ & 4.9 & $40.34 \pm 1.48$ & 3.7 & $40.22 \pm 0.40$ & 1.0 \\
\hline & 800 & $799.61 \pm 7.41$ & 0.9 & $796.41 \pm 5.25$ & 0.7 & $808.06 \pm 2.49$ & 0.3 & $801.34 \pm 5.26$ & 0.7 \\
\hline
\end{tabular}


Table 5. Pharmacokinetic parameters of 12 analytes after oral administration of Epimedium extract $(n=6)$

\begin{tabular}{|c|c|c|c|c|c|c|c|c|c|}
\hline Compounds & $T_{\max 1}(\mathrm{~h})$ & $T_{\max 2}(\mathrm{~h})$ & $C_{\max 1}(\mathrm{ng} / \mathrm{mL})$ & $C_{\max 2}(\mathrm{ng} / \mathrm{mL})$ & $t_{1 / 2}(\mathrm{~h})$ & $A U C_{(0-t n)}(\mathrm{h} \cdot \mathrm{ng} / \mathrm{mL})$ & $A U C_{(0-\infty)}(\mathrm{h} \cdot \mathrm{ng} / \mathrm{mL})$ & $M R T_{(0-t n)}(\mathrm{h})$ & $M R T_{(0-\infty)}(\mathrm{h})$ \\
\hline baohuoside II & $0.18 \pm 0.03$ & $10.67 \pm 1.03$ & $6.87 \pm 4.58$ & $29.17 \pm 7.70$ & $6.93 \pm 2.94$ & $245.95 \pm 133.39$ & $354.89 \pm 143.76$ & $11.47 \pm 1.99$ & $16.50 \pm 3.06$ \\
\hline baohuoside I & $0.38 \pm 0.14$ & $10.00 \pm 1.79$ & $65.20 \pm 33.56$ & $170.73 \pm 66.97$ & $13.25 \pm 4.80$ & $1448.99 \pm 615.56$ & $1675.75 \pm 598.59$ & $10.81 \pm 1.21$ & $15.68 \pm 4.10$ \\
\hline sagittatoside A & $0.18 \pm 0.03$ & $11.67 \pm 0.82$ & $20.35 \pm 10.26$ & $203.50 \pm 97.03$ & $13.81 \pm 7.09$ & $1614.13 \pm 348.72$ & $1782.28 \pm 351.94$ & $12.02 \pm 0.71$ & $14.26 \pm 2.00$ \\
\hline sagittatoside $B$ & $0.20 \pm 0.04$ & $11.00 \pm 1.67$ & $19.38 \pm 9.79$ & $112.97 \pm 44.50$ & $9.38 \pm 2.11$ & $853.80 \pm 171.13$ & $1036.71 \pm 201.70$ & $11.67 \pm 1.17$ & $14.89 \pm 2.09$ \\
\hline epimedin A & $0.22 \pm 0.04$ & $9.33 \pm 0.94$ & $82.79 \pm 7.61$ & $59.34 \pm 26.24$ & $12.50 \pm 6.85$ & $550.27 \pm 199.35$ & $600.14 \pm 218.86$ & $10.82 \pm 2.33$ & $14.06 \pm 3.98$ \\
\hline epimedin B & $0.19 \pm 0.07$ & $7.67 \pm 0.75$ & $316.73 \pm 59.06$ & $129.90 \pm 77.04$ & $3.64 \pm 3.68$ & $945.89 \pm 250.22$ & $1066.50 \pm 212.44$ & $6.98 \pm 1.14$ & $8.37 \pm 2.39$ \\
\hline epimedin C & $0.22 \pm 0.04$ & $7.67 \pm 0.75$ & $135.22 \pm 31.26$ & $103.20 \pm 33.10$ & $17.85 \pm 8.23$ & $850.40 \pm 268.63$ & $919.35 \pm 214.91$ & $9.33 \pm 2.34$ & $12.54 \pm 2.59$ \\
\hline magnoflorine & $0.18 \pm 0.03$ & $10.67 \pm 1.63$ & $411.40 \pm 110.77$ & $275.67 \pm 89.36$ & $17.77 \pm 8.25$ & $3676.19 \pm 874.51$ & $4003.32 \pm 969.59$ & $9.86 \pm 0.75$ & $12.52 \pm 1.72$ \\
\hline chlorogenic acid & $0.19 \pm 0.05$ & & $540.28 \pm 225.18$ & & $0.45 \pm 0.30$ & $879.68 \pm 365.08$ & $886.48 \pm 376.98$ & $3.85 \pm 1.28$ & $3.91 \pm 1.32$ \\
\hline neochlorogenic acid & $0.19 \pm 0.05$ & & $205.96 \pm 75.12$ & & $0.64 \pm 0.27$ & $263.82 \pm 68.24$ & $268.58 \pm 70.86$ & $3.18 \pm 1.57$ & $3.25 \pm 1.97$ \\
\hline cryptochlorogenic acid & $0.19 \pm 0.05$ & & $269.77 \pm 114.07$ & & $0.53 \pm 0.24$ & $397.96 \pm 170.58$ & $404.11 \pm 168.92$ & $3.75 \pm 1.91$ & $3.82 \pm 2.48$ \\
\hline icariin & $0.16 \pm 0.05$ & & $350.28 \pm 195.96$ & & $0.41 \pm 0.19$ & $322.75 \pm 204.68$ & $324.78 \pm 209.27$ & $2.71 \pm 0.79$ & $2.75 \pm 0.97$ \\
\hline
\end{tabular}



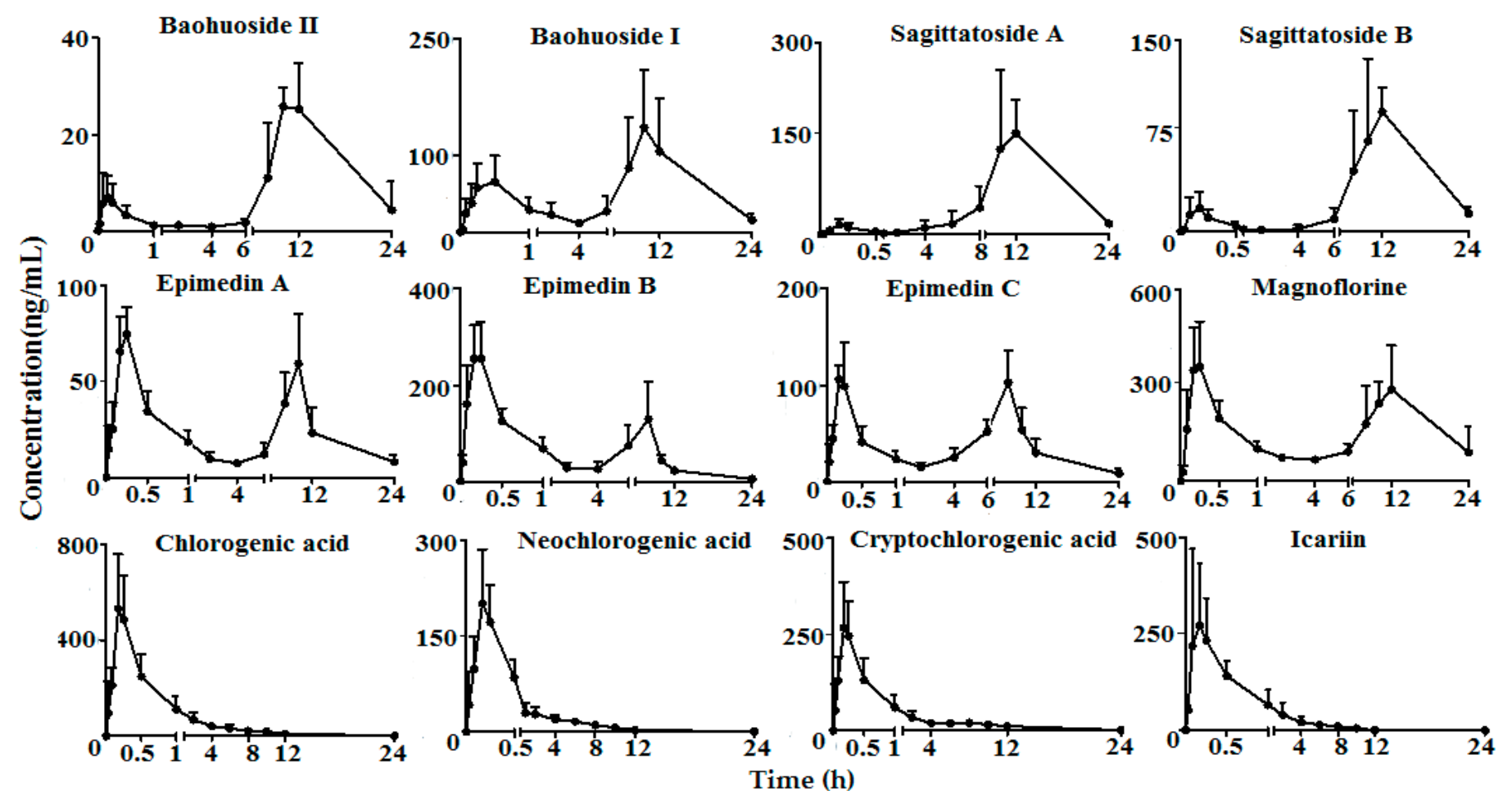

Figure 2. Mean plasma concentration-time curves of baohuoside II, baohuoside 1, sagittatoside A, sagittatoside B, epimedin A, epimedin B, epimedin C, magnoflorine, chlorogenic acid, neochlorogenic acid, cryptochlorogenic acid and icariin in six rats after oral administration of Epimedium extract (mean \pm SD). 


\section{Experimental}

\subsection{Chemicals and Reagents}

Methanol and acetonitrile (chromatographic grade) were obtained from Fisher Scientific (Fair Lawn, NJ, USA). The standard compounds of baohuoside I, baohuoside II, sagittatoside A, sagittatoside $B$, magnoflorine, epimedin $A$, epimedin $B$, epimedin $C$, chlorogenic acid, neochlorogenic acid, cryptochlorogenic acid, icariin and liquiritin (internal standard, IS) (purity $\geq 98 \%$ ) were provided by YIFANG S\&T Co. Ltd. (Tianjin, China). The chemical structures of the compounds are shown in Figure 3. Formic acid (chromatographic grade) was obtained from ROE (St. Louis, MO, USA). Deionized water was supplied by an Alpha-Q water purification system (Bedford, MA, USA).

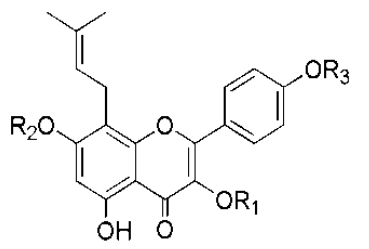

\begin{tabular}{rccc} 
& $\mathrm{R}_{1}$ & $\mathrm{R}_{2}$ & $\mathrm{R}_{3}$ \\
\cline { 2 - 4 } baohuoside II: & $\mathrm{Rha}$ & $\mathrm{H}$ & $\mathrm{H}$ \\
baohuoside I: & $\mathrm{Rha}$ & $\mathrm{H}$ & $\mathrm{CH}_{3}$ \\
sagittatoside A: & $\mathrm{Glc} \frac{12}{2} \mathrm{Rha}$ & $\mathrm{H}$ & $\mathrm{CH}_{3}$ \\
sagittatoside B: & Xyl $\frac{12}{2} \mathrm{Rha}$ & $\mathrm{H}$ & $\mathrm{CH}_{3}$ \\
epimedin A: & Glc $\frac{12}{2} \mathrm{Rha}$ & $\mathrm{Glc}$ & $\mathrm{CH}_{3}$ \\
epimedin B: & Xyl $\frac{12}{2} \mathrm{Rha}$ & $\mathrm{Glc}$ & $\mathrm{CH}_{3}$ \\
epimedin C: & Rha $\frac{12}{2} \mathrm{Rha}$ & $\mathrm{Glc}$ & $\mathrm{CH}_{3}$ \\
Icariin: & Rha & $\mathrm{Glc}$ & $\mathrm{CH}_{3}$ \\
\cline { 2 - 4 }
\end{tabular}<smiles>O=C(/C=C/c1ccc(O)c(O)c1)O[C-]1CC(O)(C(=O)O)CC(O)[C@H]1O</smiles>

chlorogenic acid

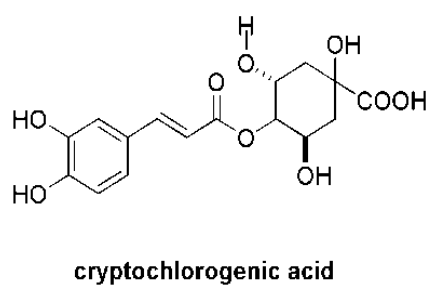<smiles>O=C(/C=C/c1ccc(O)c(O)c1)O[C@H]1C[C@@](O)(C(=O)O)C[C@H](O)[C@H]1O</smiles>

neochlorogenic acid<smiles>COc1ccc2c(c1O)-c1c(O)c(OC)cc3c1C(C2)N(C)CC3</smiles>

magnoflorine

Figure 3. The chemical structures of the 12 compounds.

\subsection{HPLC-MS/MS Analysis}

An Agilent 1200 Series HPLC system, which consisted of a binary pump (G1312A), an auto-sampler (G1367B), an online degasser unit (G1322A), along with temperature-controlled column compartment (G1316A), was used for the analysis. The twelve compounds and IS were separated on a CORTECS ${ }^{\circledR} \mathrm{C} 18$ column $(4.6 \mathrm{~mm} \times 150 \mathrm{~mm}, 2.7 \mu \mathrm{m})$ maintained at $30{ }^{\circ} \mathrm{C}$ with the mobile phase consisted of acetonitrile $(\mathrm{A})$ and $0.1 \%(\mathrm{v} / \mathrm{v})$ formic acid $(\mathrm{B})$. The flow rate was $0.3 \mathrm{~mL} / \mathrm{min}$. The gradient elution was as follows: 0-4 min, 20-50\% A; 4-5 min, 50-60\% A; 5-15 $\min , 60-70 \%$ A. The injection volume was $5 \mu \mathrm{L}$.

The tandem mass spectrometry was performed on a triple-quadrupole mass spectrometer equipped with an electrospray ionization (ESI) source (Agilent G6430A system). Nitrogen gas served as the nebulizer, drying, and collision gas. Analytes were monitored by SRM in negative mode with the electrospray ionization parameters optimized as follows: ion spray voltage of $-4000 \mathrm{~V}$, capillary temperature of $350{ }^{\circ} \mathrm{C}$, auxiliary nitrogen pressure of 20 psi, drying gas flow of $9 \mathrm{~L} / \mathrm{min}$. The mass spectrometric parameters of precursor ion, product ion, collision energy (CE) and fragmentor (Frag) for analytes are listed in Table 6. Both the collision energy and the fragmentor were optimized for each compound by infusion of the standard solutions of methanol to mass spectrometry directly. The peak in sample was recognized as the target compound if the retention time, precursor ion, and product ion are same as the stand. For each analyte, the most abundant product ion was chosen for quantification. All analytes were quantified using the 6-point calibration curve. The peak areas were used for quantification following an internal algorithm. For each batch of samples processed and analyzed, the determined concentration of each compound in the QC samples, as quantified by the standard curves, was required to fall within $\pm 15 \%$ of the known concentration for the data to be included in the final analysis. 
Table 6. Mass spectra properties of 12 analytes and IS.

\begin{tabular}{ccccc}
\hline Compounds & Precursor Ion $(\boldsymbol{m} / \mathbf{z})$ & Product Ion $(\boldsymbol{m} / \mathbf{z})$ & Frag. $($ V) & C.E. $($ V) \\
\hline baohuoside II & 499.2 & 353.0 & 140 & 20 \\
baohuoside I & 513.2 & 366.0 & 140 & 20 \\
sagittatoside A & 675.2 & 367.0 & 145 & 32 \\
sagittatoside B & 645.2 & 366.2 & 145 & 30 \\
epimedin A & 675.1 & 365.8 & 130 & 32 \\
epimedin B & 645.1 & 365.6 & 145 & 30 \\
epimedin C & 659.2 & 365.7 & 145 & 30 \\
magnoflorine & 340.1 & 310.1 & 145 & 22 \\
chlorogenic acid & 353.0 & 191.0 & 90 & 10 \\
neochlorogenic acid & 352.9 & 191.0 & 115 & 10 \\
cryptochlorogenic acid & 353.1 & 172.9 & 100 & 10 \\
icariin & 721.0 & 513.2 & 145 & 10 \\
liquiritin (IS) & 417.1 & 255.0 & 145 & 13 \\
\hline
\end{tabular}

\subsection{Preparation of Extract from Epimedium}

For the preparation of Epimedium extract, $1000 \mathrm{~g}$ of Epimedium was extracted twice by refluxing with $16 \mathrm{~L}$ of $70 \%(v / v)$ ethanol for $1.5 \mathrm{~h}$ each time. The extraction solutions were filtered and combined. Then, the Epimedium extract was obtained by evaporating the combined solutions to dryness under reduced pressure. To calculate the dosage of administration, the contents of 12 analytes were determined by the analytical method described in Section 3.2. The contents of baohuoside I, baohuoside II, sagittatoside A, sagittatoside B, magnoflorine, epimedin A, epimedin B, epimedin C, chlorogenic acid, neochlorogenic acid, cryptochlorogenic acid and icariin in Epimedium extract were $2.75,0.61,0.80,1.34,7.32,3.22,3.50,3.54,3.59,0.61,2.14$ and $13.81 \mathrm{mg} / \mathrm{g}$, respectively.

\subsection{Working Solutions}

To make the stock solutions, the standards of 12 analytes were separately weighed and dissolved in methanol. The appropriate amount of the 12 stock solutions were mixed and diluted with methanol to get a primary mixed working solution containing $1 \mu \mathrm{g} / \mathrm{mL}$ of baohuoside II; $2 \mu \mathrm{g} / \mathrm{mL}$ of baohuoside $\mathrm{I}$, sagittatoside A, sagittatoside B, epimedin A, epimedin B and epimedin C; $10 \mu \mathrm{g} / \mathrm{mL}$ of chlorogenic acid; $4 \mu \mathrm{g} / \mathrm{mL}$ of magnoflorine, neochlorogenic acid, cryptochlorogenic acid and icariin, respectively. Then, the primary mixed working solution was diluted with methanol to obtain a series of working solutions at appropriate concentrations. Liquiritin was prepared in methanol as IS solution at $1 \mu \mathrm{g} / \mathrm{mL}$.

The calibration curves were prepared by adding appropriate amounts of the working solutions to blank rats plasma $(100 \mu \mathrm{L})$ with $20 \mu \mathrm{L}$ of IS, yielding a series of concentrations at $1,2,10,50$, 100, $200 \mathrm{ng} / \mathrm{mL}$ for baohuoside II; 2, 4, 20, 100, 200, $400 \mathrm{ng} / \mathrm{mL}$ for baohuoside I, sagittatoside A, sagittatoside B, epimedin A, epimedin B and epimedin C; 10, 20, 100, 500, 1000, $2000 \mathrm{ng} / \mathrm{mL}$ for chlorogenic acid; 4, 8, 40, 200, 400, $800 \mathrm{ng} / \mathrm{mL}$ for magnoflorine, neochlorogenic acid, cryptochlorogenic acid and icariin.

Quality control (QC) samples containing 12 analytes at low, medium and high concentrations of 1 , 10, $200 \mathrm{ng} / \mathrm{mL}$ for baohuoside II; 2, 20, $400 \mathrm{ng} / \mathrm{mL}$ for baohuoside I, sagittatoside A, sagittatoside B, epimedin A, epimedin B and epimedin C; 10, 100, $2000 \mathrm{ng} / \mathrm{mL}$ for chlorogenic acid; 4, 40, $800 \mathrm{ng} / \mathrm{mL}$ for magnoflorine, neochlorogenic acid, cryptochlorogenic acid and icariin were prepared in the same manner. All the working solutions and QC samples were stored at $4{ }^{\circ} \mathrm{C}$.

\subsection{Sample Preparation}

The plasma sample $(100 \mu \mathrm{L})$ was mixed with $20 \mu \mathrm{L}$ of methanol (volume of the corresponding working solution for calibration curve and QC sample), $20 \mu \mathrm{L}$ of IS $(1 \mu \mathrm{g} / \mathrm{mL}), 20 \mu \mathrm{L}$ of formic acid and $400 \mu \mathrm{L}$ of acetonitrile. Then, the mixture was vortexed for $3 \mathrm{~min}$ and centrifuged at $14,000 \mathrm{~g}$ for $10 \mathrm{~min}$. The upper organic phase $(370 \mu \mathrm{L})$ was removed to a clean $1.5 \mathrm{~mL}$ Eppendorf tube (EP tube) 
and evaporated to dryness under a gentle nitrogen stream. After that, the residue was dissolved in $100 \mu \mathrm{L}$ methanol, vortexed for $3 \mathrm{~min}$ and centrifuged at $14,000 \mathrm{~g}$ for another $10 \mathrm{~min}$. Finally, an aliquot of $5 \mu \mathrm{L}$ of the upper organic layer was injected into the HPLC-MS/MS system for analysis.

\subsection{Method Validation}

\subsubsection{Selectivity}

The selectivity was carried out by comparing the blank plasma samples from six different rats, blank plasma samples added with analytes and IS, and post-dosing plasma samples to evaluate any potentially interfering substances.

\subsubsection{Calibration Curve}

The calibration curves were prepared by assaying standard plasma samples at six concentrations as described in Section 3.4. The linearity of each calibration curve was determined by plotting the ratio of the chromatographic peaks area (analytes/IS) versus the concentration of these analytes with a weighted $\left(1 / \mathrm{x}^{2}\right)$ least square linear regression model. Lower limit of quantification (LLOQ) was the lowest concentration of analytes which can achieve a reliable accuracy and precision with signal-noise ratio $(\mathrm{S} / \mathrm{N})$ of about 10 .

\subsubsection{Precision and Accuracy}

The intra- and inter-day precision and accuracy were measured by analysis of six replicates QC samples at low, medium and high concentrations (1, 10, $200 \mathrm{ng} / \mathrm{mL}$ for baohuoside II; 2, 20, $400 \mathrm{ng} / \mathrm{mL}$ for baohuoside I, sagittatoside A, sagittatoside B, epimedin A, epimedin B and epimedin C; 10, 100, $2000 \mathrm{ng} / \mathrm{mL}$ for chlorogenic acid; 4, 40, $800 \mathrm{ng} / \mathrm{mL}$ for magnoflorine, neochlorogenic acid, cryptochlorogenic acid and icariin) on three consecutive days. Precision was expressed as the RSD, which should not exceed 15\% (except for LLOQ less than 20\%). The accuracy expressed as the relative error (RE) was assessed by comparing the measured concentration with its true value and accepted within $\pm 15 \%$ (except for LLOQ within $\pm 20 \%$ ).

\subsubsection{Extraction Recovery and Matrix Effect}

The extraction recovery was determined by comparing the peak areas of six replicates QC samples at three concentrations with post-extraction spiked samples. The matrix effect was assessed by comparing the peak areas of post-extraction spiked samples with working solution at the same concentration on three QC levels.

\subsubsection{Stability}

The stability of all analytes in rat plasma was investigated by testing QC samples at three concentrations under various conditions as followed: storage at room temperature for $2 \mathrm{~h}$, in auto-sampler for $12 \mathrm{~h}$, at $-70^{\circ} \mathrm{C}$ for 7 days and three freeze-thaw cycles. The RSDs of the stability should be within $15 \%$.

\subsection{Pharmacokinetic Studies}

Male Sprague-Dawley rats $(220 \pm 10) \mathrm{g}$ were obtained from the Beijing HUAFUKANG Bioscience Co., Inc. (Beijing, China). Six male rats were acclimatized to the facilities for a week and fasted for $12 \mathrm{~h}$, but allowed free access to water, before dosing. The Epimedium extract was suspended in $0.5 \%$ carboxymethyl cellulose sodium (CMC-Na) aqueous solution and given to each rat by oral administration at a single dose of $10 \mathrm{~g} / \mathrm{kg}$. Blood samples $(250 \mu \mathrm{L})$ were collected from the fossa orbitalis of rats at 0 (before dosing), $0.03,0.08,0.17,0.25,0.5,1,2,4,6,8,10,12$ and $24 \mathrm{~h}$ after dosing. Then, the blood samples were centrifuged at $6000 \mathrm{~g}$ for $10 \mathrm{~min}$ to obtain the plasma, and the plasma was 
removed to another EP tube and frozen at $-70{ }^{\circ} \mathrm{C}$ until analysis. The rat plasma concentration-time data of 12 analytes were computed by the software "Drug and Statistics 1.0" (DAS 1.0)

\section{Conclusions}

In this research, a rapid and reliable HPLC-MS/MS method was established to simultaneously determine twelve bioactive compounds (baohuoside I, baohuoside II, sagittatoside A, sagittatoside B, magnoflorine, epimedin A, epimedin B, epimedin C, chlorogenic acid, neochlorogenic acid, cryptochlorogenic acid and icariin) in rat plasma. The validated method was successfully applied to the pharmacokinetic study of twelve bioactive compounds after oral administration of Epimedium extract in rats. Furthermore, this is also the first pharmacokinetic study of baohuoside II and sagittatoside A. The pharmacokinetic study may contribute to the clinical usage of Epimedium.

Author Contributions: J.H. and X.G. designed the research; M.S., Y.Y. and J.W. wrote the manuscript and performed the experimental work; H.O. analyzed the data; X.C. and Y.C. perfected language. All authors discussed, edited and approved the final version.

Funding: This study was supported by National Natural Science Foundation of China $(81673824,81503457)$, Tianjin Municipal Education Commission Research Project (2017KJ139), Important Drug Develop of MOST, China (2015ZX09501004-003-004).

Conflicts of Interest: The authors declare no conflict of interest.

\section{References}

1. Hang, D.X.; Yang, J.; Lu, X.M.; Deng, Y.; Xiong, Z.L.; Li, F.M. An integrated plasma and urinary metabonomic study using UHPL-MS: intervention effects of Epimedium koreanum on 'Kidney-Yang Deficiency syndrome' rats. J. Pharm. Biomed. Anal. 2013, 76, 200-206. [CrossRef] [PubMed]

2. Zhang, H.F.; Zhang, X.; Yang, X.H.; Qiu, N.X.; Wang, Y.; Wang, Z.Z. Microwave assisted extraction of flavonoids from cultivated Epimedium sagittatum: extraction yield and mechanism, antioxidant activity and chemical composition. Ind. Crop. Prod. 2013, 50, 857-865. [CrossRef]

3. Sze, S.C.; Tong, Y.; Ng, T.B.; Cheng, C.L.; Cheung, H.P. Herba Epimedii: antioxidative properties and its medical implications. Molecules 2010, 15, 7861-7870. [CrossRef] [PubMed]

4. Jiang, J.; Song, J.; Jia, X.B. Phytochemistry and ethnopharmacology of Epimedium L. species. Chin. Herb. Med. 2015, 7, 204-222. [CrossRef]

5. Pan, Y.; Kong, L.D.; Xia, X.; Zhang, W.Y.; Xia, Z.H.; Jiang, F.X. Antidepressant-like effect of icariin and its possible mechanism in rats. Pharmacol. Biochem. Behav. 2005, 82, 686-694. [CrossRef] [PubMed]

6. Wang, Z.Q.; Lou, Y.J. Proliferation-stimulating effects of icaritin and desmethylicaritin in MCF-7 cells. Eur. J. Pharmacol. 2004, 504, 147-153. [CrossRef] [PubMed]

7. Liu, Y.Q.; Han, X.F.; Liu, T.G.; Cheng, M.C.; Xiao, H.B. A cell-based model of bone remodeling for identifying activity of icarrin in the treatment of osteoporosis. Biotechnol. Lett. 2015, 37, 219-226. [CrossRef] [PubMed]

8. Ma, H.P.; He, X.R.; Yang, Y.; Li, M.X.; Hao, D.J.; Jia, Z.P. The genus Epimedium: an ethnopharmacological and phytochemical review. J. Ethnopharmacol. 2011, 134, 519-541. [CrossRef] [PubMed]

9. Wang, Y.A.; Guo, Z.M.; Jin, Y.; Zhang, X.L.; Wang, L.; Xue, X.Y.; Liang, X.M. Identification of prenyl flavonoid glycosides and phenolic acids in Epimedium koreanum Nakai by Q-TOF-MS combined with selective enrichment on click oligo (ethylene glycol) column. J. Pharm. Biomed. Anal. 2010, 51, 606-616. [CrossRef] [PubMed]

10. Zulfiqar, F.; Khan, S.I.; Ross, S.A.; Ali, Z.; Khan, I.A. Prenylated flavonol glycosides from Epimedium grandiflorum: Cytotoxicity and evaluation against inflammation and metabolic disorder. Phytochemistry 2017, 20, 160-167. [CrossRef]

11. Zhang, D.W.; Cheng, Y.; Wang, N.L.; Zhang, J.C.; Yang, M.S.; Yao, X.S. Effects of total flavonoids and flavonol glycosides from Epimedium koreanum Nakai on the proliferation and differentiation of primary osteoblasts. Phytomedicine 2008, 15, 55-61. [CrossRef] [PubMed]

12. Yao, Z.H.; Liu, M.Y.; Dai, Y.; Zhang, Y.; Qin, Z.F.; Tu, F.J.; Yao, X.S. Metabolism of Epimedium-derived flavonoid glycosides in intestinal flora of rabbits and its inhibition by gluconolactone. Chin. J. Nat. Med. 2011, 9, 461-465. [CrossRef] 
13. Krych, J.L. Gebicka. Catalase is inhibited by flavonoids. Int. J. Biol. Macromol. 2013, 58, 148-153. [CrossRef] [PubMed]

14. Hartmann, T. From waste products to ecochemicals: Fifty years research of plant secondary metabolism. Phytochemistry. 2007, 68, 2831-2846. [CrossRef] [PubMed]

15. Patel, M.B.; Mishra, S.M. Magnoflorine from Tinospora cordifolia stem inhibits $\alpha$-glucosidase and is antiglycemic in rats. J. Funct. Foods 2012, 4, 79-86. [CrossRef]

16. Wang, C.C.; Feng, L.; Su, J.Y.; Cui, L.; Liu, D.J.; Yan, C.L.; Ding, C.L.; Tian, X.B.; Jia, X.B. Polysaccharides from Epimedium koreanum Nakai with immunomodulatory activity and inhibitory effect on tumor growth in LLC-bearing rats. J. Ethnopharmacol. 2017, 207, 8-18. [CrossRef] [PubMed]

17. Cheng, H.R.; Feng, S.L.; Shen, S.A.; Zhang, L.; Yang, R.W.; Zhou, Y.H.; Ding, C.B. Extraction, antioxidant and antimicrobial activities of Epimedium acuminatum Franch. polysaccharide. Carbohyd. Polym. 2013, 96, 101-108. [CrossRef] [PubMed]

18. Wong, S.P.; Shen, P.; Lee, L.; Li, J.; Yong, E.L. Pharmacokinetics of prenylflavonoids and correlations with the dynamics of estrogen action in sera following ingestion of a standardized Epimedium extract. J. Pharm. Biomed. Anal. 2009, 50, 216-223. [CrossRef] [PubMed]

19. Wu, C.S.; Zhang, J.L.; Zhou, T.H.; Guo, B.L.; Wang, Y.L.; Hou, J.F. Simultaneous determination of seven flavonoids in dog plasma by ultra-performance liquid chromatography-tandem mass spectrometry and its application to a bioequivalence study of bioactive components in Herba Epimedii and Er-Xian Decoctio. J. Pharm. Biomed. Anal. 2011, 54, 186-191. [CrossRef] [PubMed]

20. Tong, L.; Wan, M.X.; Zhang, L.H.; Zhu, Y.H.; Sun, H.; Bi, K.S. Simultaneous determination of baicalin wogonoside, baicalein, wogonin, oroxylin A and chrysin of Radix scutellariae extract in rat plasma by liquid chromatography tandem mass spectrometry. J. Pharm. Biomed. Anal. 2012, 70, 6-12. [CrossRef] [PubMed]

21. Qi, W.; Zhao, T.; Yang, W.W.; Wang, G.H.; Yu, H.; Zhao, H.X.; Yang, C.; Sun, L.X. Comparative pharmacokinetics of chlorogenic acid after oral administration in rats. J. Pharm. Anal. 2011, 4, 270-274. [CrossRef] [PubMed]

22. Shen, P.; Wong, S.P.; Li, J.; Yong, E.L. Simple and sensitive liquid chromatography-tandem mass spectrometry assay for simultaneous measurement of five Epimedium prenylflavonoids in rat sera. J. Chromatogr. B 2009, 877, 71-78. [CrossRef] [PubMed]

Sample Availability: The extracts of Epimedium are available from the authors.

(C) 2018 by the authors. Licensee MDPI, Basel, Switzerland. This article is an open access article distributed under the terms and conditions of the Creative Commons Attribution (CC BY) license (http://creativecommons.org/licenses/by/4.0/). 